\title{
On the Efficiency of Interference Coordination Schemes in Emerging Cellular Wireless Networks
}

\author{
Mehrdad Shariat, Atta Ul Quddus, Rahim Tafazolli \\ Mobile VCE Research Group, Centre for Communication Systems Research \\ University of Surrey \\ Guildford, GU2 7XH, Surrey, United Kingdom \\ \{m.shariat, a.quddus and r.tafazolli\}@surrey.ac.uk
}

\begin{abstract}
In this paper, the efficiencies of different interference coordination schemes are evaluated for emerging wireless networks and the possible impact on intra-cell scheduling is studied through extensive simulations. The results show that pure fractional frequency reuse can provide similar improvement in the cell-edge throughput compared to power coordinated counterpart at a less cost in terms of overall throughput. Moreover, it can provide fairer distribution of throughput in both central as well as cell-edge areas. However, this scheme can not mange asymmetrical changes in the distribution of users across different cells in the entire system. As a result, a power coordination mechanism would be still necessary on top of such flexible frequency reuse schemes.
\end{abstract}

Index Terms- Efficiency, Interference management, OFDMA, Scheduling

\section{INTRODUCTION}

During the past decade, multi-carrier communications has gained a strong support in both academia and industry due to its inherent robustness against frequency selectivity. Thus, Orthogonal Frequency Division Multiplexing (OFDM) and its variants seem to be a dominant platform for future broadband wireless technologies [1]. The migration towards OFDM provides important opportunities in scheduling that are quite promising. This multiplexing policy enables allocation of distinct sub-channels in frequency domain to different users per time slot, i.e. Orthogonal Frequency Division multiple Access (OFDMA) that brings finer resolution of resource allocation through two-dimensional scheduling in time-frequency domain; however, unfortunately cellular systems are interference-limited and this factor can significantly affect the efficiency of OFDMA schedulers at intra-cell scale. As a result, interference management schemes are crucial to control the inter-cell interference to alleviate this issue. Each interference management policy has a direct impact on the efficiency of intra-cell scheduling algorithm in terms of throughput, fairness as well as coverage. In this direction, extensive study is required to adopt the best policy according to the system requirements as well as practical constraints. In general, such schemes can be categorized into three major approaches, i.e. interference randomization, interference cancellation and interference coordination [2]. Randomization schemes including frequency hopping and cell-specific scrambling [3] are useful to average out inter-cell interference. However, these methods are suitable to exploit frequency diversity and can not fit into the OFDMA-based scheduling particularly in full load scenarios. Cancellation schemes, on the other hand, such as interleaved division multiple access (IDMA), are getting growingly popular. However, complexity in higher orders of bandwidth particularly in the downlink is still an issue that requires further investigation [4].

In this paper, our major focus is on possible inter-cell coordination schemes on the downlink side to manage the interference at intra-cell scale. First, potential policies are introduced based on the literature. Thereafter, the developed schemes are evaluated and compared in an OFDMA context to study their effects on the overall system efficiency. Considering the outcome results the advantages and disadvantages of different methods are pointed out

Following this brief introduction, section II, describes some of the state-of-art interference coordination schemes. In section III, a simulation methodology is introduced to verify the developed concepts. Here, proportional fair (PF) scheduler is evaluated in a multi-cell and multi-user environment as the benchmark algorithm. Thereafter, the efficiencies of different interference coordination schemes are compared to this benchmark scenario and advantages and disadvantages are pointed out. Finally in section IV, future issues and challenges are identified.

\section{STATE-OF-THE-ART INTERFERENCE COORDINATION SCHEMES}

Interference coordination schemes try to alleviate interference through defining an inter-cell interference controlling mechanism. Such controlling mechanisms can happen either in static or dynamic manner.

Considering, static coordination, non-overlapping resource units in frequency-time domain must be allocated to neighboring cells to avoid interference. Unfortunately, dividing the resources among adjacent interfering cells will reduce the diversity of available resources per cell and does not drive the potential throughput efficiency of channel-aware scheduling algorithms. Alternatively, fractional frequency reuse (FFR) [5] can provide more feasible solution. This method exploits the non-balanced property of interference to provide more resource diversity in the central area of each cell compared to complete 
partitioning of resources. This is mainly due to the fact that interference is strongest at the cell-edge area and users in celledge vicinity are more likely to get affected. Based on this method, each cell is divided into central and cell-edge area. Users in the cell-edge area are provided with a fraction of total resources but they experience a lower level of interference whereas central area users can exploit the diversity of all the remainder of resources. This method enables the intra-cell schedulers to assign the resources in a fairer manner across the cell. However, fractional dividing of the resources still reduces the trunking gain ${ }^{1}$. Dynamic interference coordination, on the other hand, can potentially provide more degree of freedom for intra-cell scheduling. However, the price for such methods must be carefully considered as dynamic coordination imposes signaling among neighboring cells and inevitably leads to higher orders of delay in intra-cell scheduling. Moreover, it violates the independency of intra-cell schedulers as it defines a higher layer of management on top of intra-cell resource allocation algorithms. Nevertheless, such dynamic schemes can be limited to a small set of strong interfering cells to keep the independency and delay within acceptable thresholds.

In following section, we develop a simulation methodology and thereafter we investigate the effects of different coordination schemes on a classical scheduling algorithm.

\section{SIMULATION STUDY}

The case study is done on the downlink of a typical OFDM system consisting of wraparound 7 cells as shown in Fig. 1 to evaluate a classical scheduling algorithm in different coordination schemes. We consider a 10-user scenario in which users are located at different layers of distance from the base station. Each user is assumed to be moving on a ring with a fixed distance from the base station. In other words, each user is representative of all potential users at that distance from the base station. By this approach, effective distribution of cellthroughput in the entire cell is obtained. Without any loss of generality, the users are sorted based on their distance from the base station. Moreover, a backlogged traffic is considered and power is distributed identically on all sub-channels (fixed power allocation). The rest of simulation parameters (similar to that in [2]) are shown in Table I.

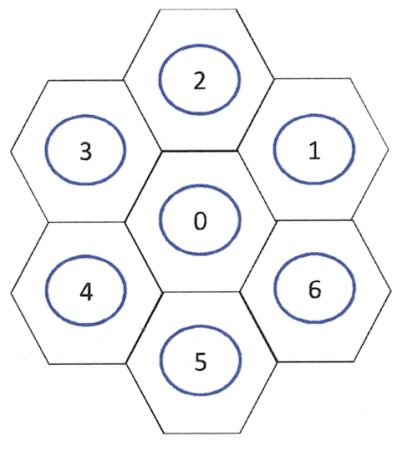

Figure 1. Employed cell grid

\footnotetext{
1 Trunking gain refers to the gain that is achieved by merging resources into a single shared resource pool.
}

\begin{tabular}{l|l}
\hline Target BER & $10 \mathrm{e}-4$ \\
No. of users $(N)$ & $10(\mathrm{In}$ central cell only) \\
Bandwidth & $5 \mathrm{MHz}$ \\
No. of sub-channels & 25 \\
No. of sub-carriers per sub-channel & 12 \\
Sub-frame duration & $0.5 \mathrm{~ms}$ \\
Channel model & $\mathrm{ITU}$ Pedestrian B \\
Path loss model & $\mathrm{L}=128.1+37.6 \mathrm{Log}(\mathrm{R}), \mathrm{R}[\mathrm{Km}]$ \\
Total BS transmission power & $43 \mathrm{dBm}$ \\
BS antenna gain & $14 \mathrm{dBi}$ \\
Modulation & $\mathrm{QPSK}, 16-\mathrm{QAM}, 64-\mathrm{QAM}$ \\
Coding rate & $1 / 6,1 / 3,1 / 2,2 / 3,3 / 4,5 / 6,1$ \\
Channel estimation & $\mathrm{Ideal}$ \\
Shadowing standard deviation & $8 \mathrm{~dB}$ \\
Shadowing correlation distance & $50 \mathrm{~m}$ \\
User speed & $3 \mathrm{Km} / \mathrm{h}$ \\
Cell Radius & $1 \mathrm{Km}$ \\
\hline
\end{tabular}

\section{A. Full interference}

In the first scenario, all resources are shared in an uncoordinated manner among the adjacent cells. This implies frequency reuse factor of one in the entire cell area or explicit interference modeling. PF algorithm is considered at intra-cell scale and the optimal candidate user for each sub-channel is matched based on a gradient search method as follows:

$$
n_{k}^{*}=\operatorname{argmax}_{n}\left(\frac{r_{n, k}}{\bar{r}_{n}}\right)
$$

Here, $n^{*}$ represents the optimal user for resource $k$ whereas $r_{n, k}$ shows the potential rate of user $n$ on the target resource. Moreover, $\overline{r_{n}}$ symbolizes the average data rate of user $n$ over the past time-slots. The averaging process happens based on a real-time exponential averaging as follows [6]:

$$
\bar{r}_{n}^{t}=(1-\alpha) \cdot \bar{r}_{n}^{t-1}+\alpha \cdot r_{n}^{t}
$$

Here, $\alpha$ is inversely proportional to the time window of scheduler and $r_{n}^{t}$ represents the aggregate throughput of user $n$ on current time slot, $t$.

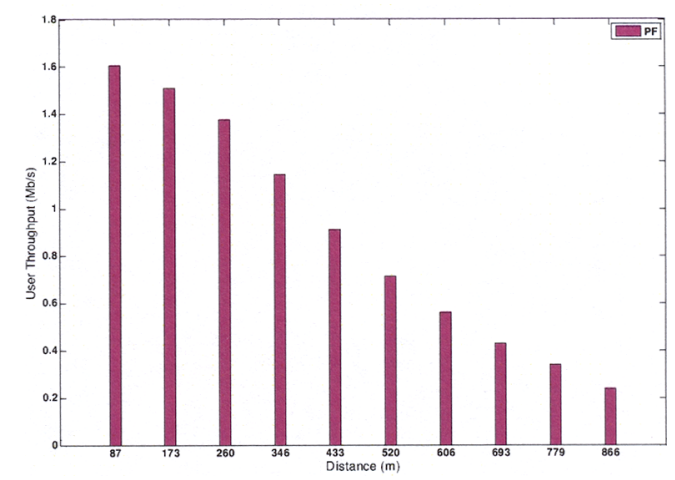

Figure 2. Performance of PF scheduler in full interference scenario 
Fig. 2 illustrates the outcome result in full interference scenario for $\alpha=0.01$. Here, the effective distribution of throughput is shown based on the distance from the base station. PF scheduler provides a reasonable compromise between fairness and cell-throughput through long term channel awareness. However, in full interference condition, this scheme suffers from low data rates in the cell-edge area due to strong co-channel interference (CCI) from the adjacent cells. This benchmark scenario clearly shows the necessity of interference management. In the following sub-sections, the above mentioned intra-cell scheduling concept is evaluated in different coordination schemes.

\section{B. Coordinated transmission of power}

Adaptive power allocation has been considered in some proposals as a complementary scheme to enhance the efficiency of resource scheduling at intra-cell scale [7]-[9]. It has been proven that adaptive power allocation can bring significant improvement in the capacity of the system in case of discrete rate adaptation [8]. However, such schemes can happen at the cost of extra complexity as well as signaling in the system [9]. Moreover, the gain diminishes by increasing the available modulation and coding schemes as the system gets closer to the continuous rate adaptation process. On the other hand, in practical scenarios boosting the power on some resources at intra-cell scale can cause strong interference on those resources in neighboring cells. In other words, improvement in one cell might turn out to be at the cost of service in adjacent cells. In this direction, fixed power allocation seems like a robust solution, that bypasses extra complexity, signaling and strong inter-cell dependencies. However, considering the problem at inter-cell scale, simple inter-cell power coordination methods can be employed to control the level of interference among the adjacent cells. One such scheme is shown in Fig. 3.

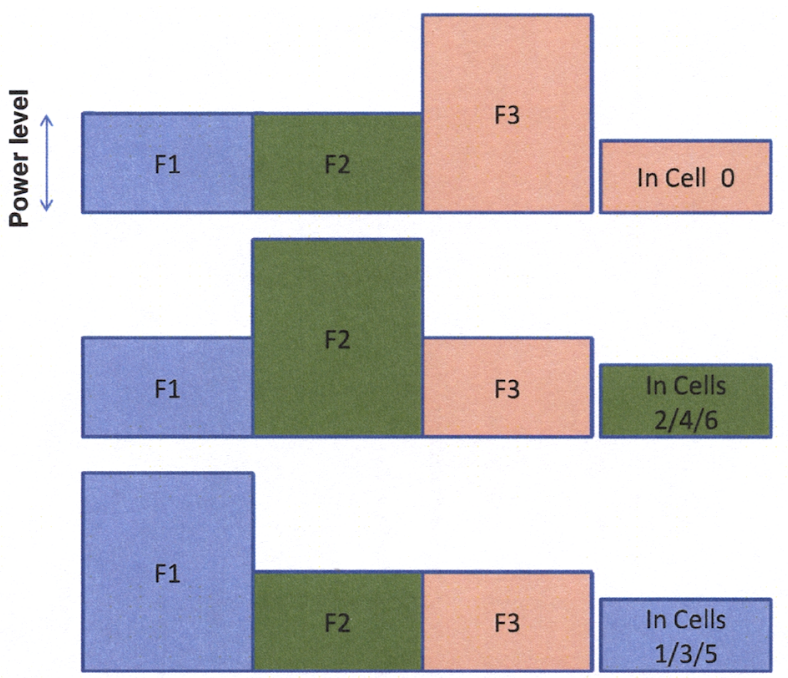

Figure 3. Inter-cell power coordination scheme across neighboring cells

Based on this coordination method, the total bandwidth is divided into three distinct bands. Each cell transmits on full power on its exclusive band, whereas it reduces the power on the other two bands. This power coordination scheme provides a platinum band per cell on lower level of interference. For example in cell $0, \mathrm{~F} 3$ provides the platinum band whereas $\mathrm{F} 1$ and $\mathrm{F} 2$ are the non-platinum bands.

In this coordination scheme, two different resource allocation procedures are possible. One approach is to employ the same benchmark allocation scheme, i.e. keeping the frequency reuse factor of one in the entire cell area to simply let the intra-cell scheduling algorithm choose the optimal resources for different users. The advantage of this method lies in the fact that the resource pool is not partitioned into separate groups for central and cell-edge areas. Fig. 4 demonstrates the results for this allocation procedure in 3 different levels of power reduction compared to the benchmark algorithm. Here $\beta$ represents the fraction of nominal power which is transmitted on two non-platinum bands per cell. As it can be seen in Table II, this simple power coordination scheme provides higher cellthroughput compared to the benchmark. However, users close to the base station take more advantage from this coordination scheme compared to the cell-edge ones. Therefore, enhancement in the cell-edge area is still marginal compared to the benchmark algorithm.

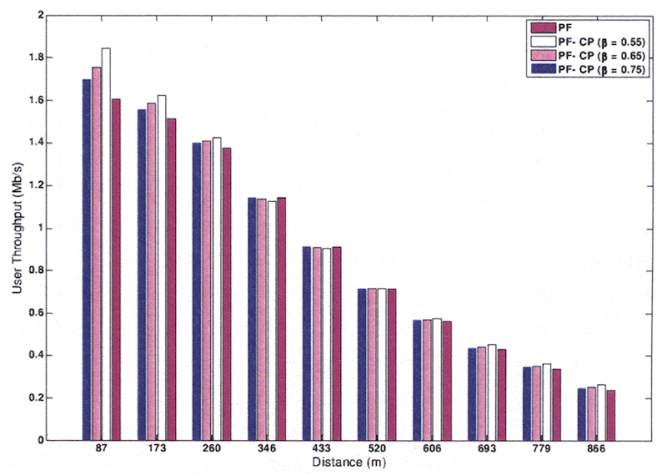

Figure 4. Performance of coordinated power (CP) compared to the benchmark.

TABLE II. COMPARING PERFORMANCE OF PF-CP TO PURE PF

\begin{tabular}{|c|c|c|c|c|}
\hline & $\begin{array}{c}\text { Central } \\
\text { T-put } \\
{[\mathbf{M b} / \mathbf{s}]}\end{array}$ & $\begin{array}{c}\text { Cell-Edge } \\
\text { T-put } \\
\text { [Mb/s] }\end{array}$ & $\begin{array}{c}\text { Overall } \\
\text { T-put } \\
{[\mathbf{M b} / \mathbf{s}]}\end{array}$ & $\begin{array}{l}\text { Fairness } \\
\text { index }^{a}\end{array}$ \\
\hline PF (pure) & 7.8205 & 1.0063 & 8.8268 & 0.7739 \\
\hline PF-CP (B: 0.55) & 8.2162 & 1.0802 & 9.2964 & 0.7577 \\
\hline PF-CP (B: 0.65) & 8.0817 & 1.0474 & 9.1291 & 0.7631 \\
\hline PF-CP (ß: 0.75) & 7.9810 & 1.0284 & 9.0094 & 0.7672 \\
\hline
\end{tabular}

Alternatively, it is possible to partition the resources between cell-edge and central area users, i.e. employing fractional frequency reuse. As a result, the platinum band is exploited by the cell-edge users whereas the remainder of resources, i.e. two other bands can be utilized by central area users. This method provides a higher level of diversity in central area compared to the cell edge but at a lower level of power. In this scenario, a criterion is required to differentiate central area users from the cell-edge ones. The criterion in our 
scenario is defined based on the distance of a user from the base station. The threshold is set to $65 \%$ of the cell radius. Thus, the central area accommodates 7 users whereas 3 far-off users are located in the cell-edge area. Resource allocation happens in three different stages. At the first stage, cell-edge users are scheduled from the platinum band of resources with lower level of interference. In the second stage, central area users are scheduled from the remainder of resources and finally, the non-utilized fraction of resources from the platinum band are reallocated to the central area as this avoids the wastage of resources and provides more diversity for the central area. Fig. 5 shows simulation results in this scenario for three different levels of power reduction. As illustrated in Table III, this scheme provides significant increase in throughput of cell-edge area. However, this improvement is at the cost of throughput in the central area. Particularly, users in the middle of cell are adversely affected in this scenario as they can barely exploit the returned fraction of platinum band at the third stage due to higher level of path loss as well as interference compared to users close to the base station.

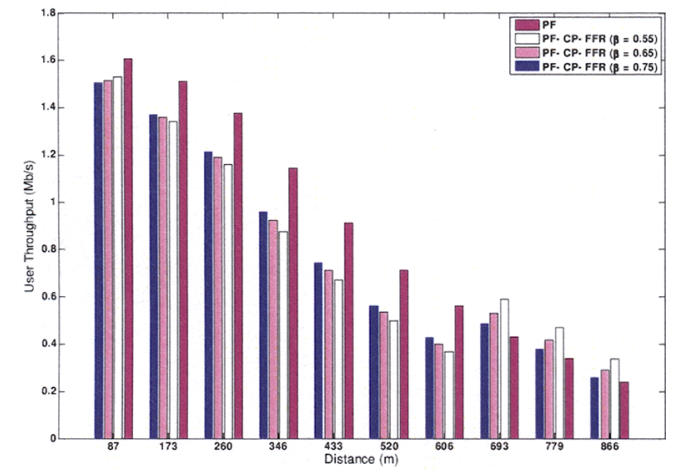

Figure 5. Performance of joint power coordination and fractional reuse (CPFFR) compared to the benchmark

TABLE III. COMPARING PERFORMANCE OF PF-CP-FFR TO PURE PF

\begin{tabular}{|c|c|c|c|c|}
\hline & $\begin{array}{c}\text { Central } \\
\text { T-put } \\
{[\mathrm{Mb} / \mathrm{s}]}\end{array}$ & $\begin{array}{c}\text { Cell-Edge } \\
\text { T-put } \\
\text { [Mb/s] }\end{array}$ & $\begin{array}{c}\text { Overall } \\
\text { T-put } \\
{[\mathbf{M b} / \mathbf{s}]}\end{array}$ & $\begin{array}{c}\text { Fairness } \\
\text { index }\end{array}$ \\
\hline PF (pure) & 7.8205 & 1.0063 & 8.8268 & 0.7739 \\
\hline PF-CP-FFR ( $\beta$ : 0.55) & 6.4457 & 1.3957 & 7.8414 & 0.7911 \\
\hline PF-CP-FFR ( $\beta$ : 0.65) & 6.6303 & 1.2372 & 7.8675 & 0.7841 \\
\hline PF-CP -FFR(B: 0.75) & 6.7741 & 1.1203 & 7.8944 & 0.7773 \\
\hline
\end{tabular}

\section{Pure Fractional Frequency Reuse}

In the allocation schemes based on inter-cell power coordination, the first-tier interference from the neighboring cells is alleviated on a fraction of bandwidth, i.e. the platinum band. However, this interference is not totally eliminated. This is due to the fact that, the coordination happens in a symmetrical pattern for all the cells. As a result, total elimination of interference on the platinum band requires reducing the power to zero in the central areas which is not a feasible solution. This clue directs us towards another approach to coordinate interference as shown in Fig. 6 that is inspired from [10].

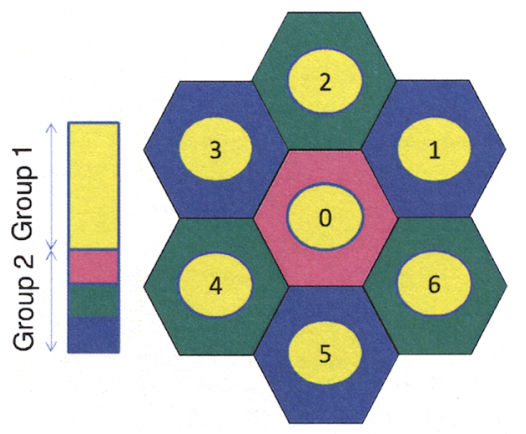

Figure 6. Pure fractional frequency reuse plan

Here, the total bandwidth is partitioned into two distinct groups. The first group is exclusively considered for the central area of all the cells whereas the second group is partitioned into three non-overlapping subgroups. Consequently, each subgroup is allocated to the cell-edge area of one of the neighboring cells similar to Fig. 6 . This flexible reuse pattern for the cell-edge area completely eliminates the first-tier interference on each allocated sub-group. Moreover, it does not require any power coordination among adjacent cells as the resources for both categories have already been separated. However, the cell-edge quota is limited compared to the power coordinated scenario as just a single subgroup is allocated to the cell-edge area per cell. Fig. 7 shows the simulation results for this new scheme in similar scenario as B where $70 \%$ of users are located in the central area and 30\% remain in the celledge.

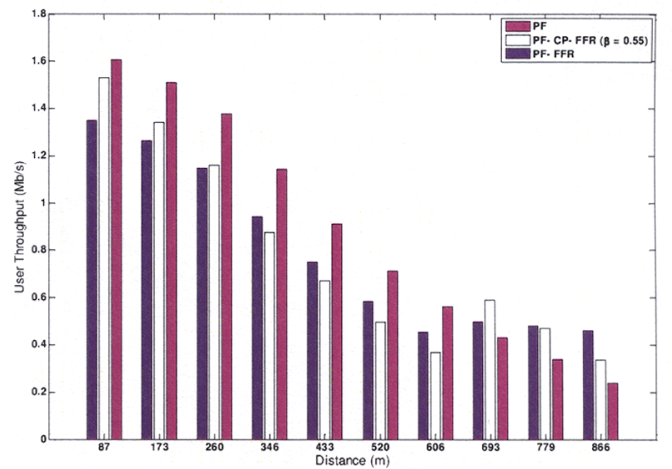

Figure 7. Performance of pure fractional frequency reuse (PF-FFR) compared to other coordination schemes

TABLE IV. COMPARING PERFORMANCE OF PF-FFR WITH PF-CP-FFR AND PURE PF

\begin{tabular}{l|cccc}
\hline & $\begin{array}{c}\text { Central } \\
\text { T-put } \\
{[\mathbf{M b} / \mathbf{s}]}\end{array}$ & $\begin{array}{c}\text { Cell-Edge } \\
\text { T-put } \\
{[\mathbf{M b} / \mathbf{s}]}\end{array}$ & $\begin{array}{c}\text { Overall } \\
\text { T-put } \\
{[\mathbf{M b} / \mathbf{s}]}\end{array}$ & $\begin{array}{c}\text { Fairness } \\
\text { index }\end{array}$ \\
\hline PF (pure) & 7.8205 & 1.0063 & 8.8268 & 0.7739 \\
PF-CP-FFR (ק: 0.55) & 6.4457 & 1.3957 & 7.8414 & 0.7911 \\
PF-FFR & 6.4851 & 1.4412 & 7.9263 & 0.8474 \\
\hline
\end{tabular}


As shown in Table IV, this coordination scheme can provide similar improvement in the cell-edge throughput at a lower level of cost compared to the power coordinated scenario. Moreover, as it can bee seen, this scheme can provide fairer distribution of throughput in both the cell-edge as well as central area. This is mainly due to the complete elimination of first-tier interference in this scheme as well as full power transmission on all the bandwidth. However, the peak achievable throughput in cell-edge area is limited compared to the power coordinated scheme as only a fraction of second group is allocated to this area. To realize the consequence of this issue, consider a scenario in which users are gradually concentrated in the cell-edge area. Inevitably, in that scenario, the distance threshold should move towards the central area, i.e. resources should be transferred from the central quota into the cell-edge pool. Unfortunately, the resources of central area belong to group one that is used in frequency reuse factor of one in all the cells. This implies that the interference level can be strong on those resources in the cell-edge area. Thus, an uncoordinated transfer of resources to the cell-edge does not bring considerable improvement, as those resources would be barely utilized in the cell-edge. This shows that although the lack of power coordination is favorable in symmetric scenarios with uniform distribution of users, such schemes may not be able to sufficiently handle the dynamic changes in the distribution of users. Therefore, undoubtedly, a power coordination layer is required on top of such flexible reuse schemes to manage more complex scenarios.

\section{CONCLUSION}

In this paper, the efficiencies of different interference coordination schemes were investigated and the possible impact on intra-cell scheduling was outlined in terms of throughput efficiency, rate-fairness and coverage. PF scheduler was employed at intra-cell scale as the benchmark algorithm. As discussed, pure fractional frequency reuse can provide similar improvement in the throughput of the cell-edge area at a less cost in overall throughput efficiency. However, asymmetry of load distribution within a cell in practical scenarios inevitably requires a higher layer of interference management on top of pure fractional frequency reuse. This can potentially impose extra signaling as well as complexity to the system. However, such schemes can be employed in a limited set of strong interfering cells to keep those overheads within acceptable thresholds.

\section{ACKNOWLEDGMENT}

The work reported in this paper has formed part of the Delivery Efficiency Core Research Program of the Virtual Centre of Excellence in Mobile \& Personal Communications, Mobile VCE, www.mobilevce.com. This research has been funded by the Industrial Companies who are Members of Mobile VCE, with additional financial support from the UK Government's Engineering \& Physical Sciences Research Council. Fully detailed technical reports on this research are available to Industrial Members of Mobile VCE.

\section{REFERENCES}

[1] D. Astely, E Dahlman, Pal Frenger, R. Ludwig, M. Meyer, S. Parkvall, P. Skillermark, and N. Wiberg, "A future radio-access framework," IEEE Journal on Selected Areas in Communications, March 2006, Vol. 24, No. 3, pp. 693-706

[2] 3GPP, "Technical specification group radio access network, physical layer aspects for Evolved UTRA," 25.814.

[3] R. Laroia, S. Uppala, and J. Li, "Designing a mobile broadband wireless access network," IEEE Signal Processing Magazine, September 2004, Vol. 21, No. 5, pp. 20-28

[4] Li Ping, Qinghua Guo, and Jun Tong, "The OFDM-IDMA approach to wireless communication systems," IEEE Wireless Communications Magazine, June 2007, Vol. 14, No. 3, pp. 18-24

[5] M. Feng, and H. Wang, "System level modeling and algorithms for a B3G system employing OFDMA," International conference on mobile technology applications and systems, November 2007, pp. 15-17

[6] H. Holma, and A. Toskala, "HSDPA/HSUPA for UMTS," JOHN WILEY \& SONS, LTD, 2006, pp. 111-113.

[7] G. Song, and Y. Li, "Cross-layer optimization for OFDM wireless networks-Part I: theoretical framework," IEEE Transactions on Wireless Communication, March 2005, Vol. 4, No. 2, pp. 614-624.

[8] G. Song, and Y. Li, "Cross-layer optimization for OFDM wireless networks-Part II: algorithm development," IEEE Transactions on Wireless Communication, March 2005, Vol. 4, No. 2, pp. 625-634.

[9] G. Song, and Y. Li, "Utility-based resource allocation and scheduling in OFDM-based wireless broadband networks," IEEE Communications Magazine, December 2005, Vol. 43, No. 12, pp. 127-134

[10] Haipeng Lei, Lei Zhang, Xin Zhang, and Dacheng Yang, "A novel multi-cell OFDMA system structure using fractional frequency reuse," IEEE 18th International Symposium on Personal, Indoor and Mobile Radio Communications, September 2007, pp. 1-5 